\title{
Correcting distortions in the infrared array camera during the cryogenic mission of the Spitzer Space Telescope
}

Carl J. Grillmair, Patrick J. Lowrance, Sean J. Carey, John R. Stauffer, James G. Ingalls, et al.

Carl J. Grillmair, Patrick J. Lowrance, Sean J. Carey, John R. Stauffer, James G. Ingalls, Jessica E. Krick, William J. Glaccum, Seppo Laine, Roberta Paladini, David L. Shupe, Schuyler D. Van Dyk, "Correcting distortions in the infrared array camera during the cryogenic mission of the Spitzer Space Telescope," Proc. SPIE 10698, Space Telescopes and Instrumentation 2018: Optical, Infrared, and Millimeter Wave, 106985D (6 July 2018); doi: $10.1117 / 12.2313706$

SPIE Event: SPIE Astronomical Telescopes + Instrumentation, 2018, Austin, Texas, United States 


\title{
Correcting Distortions in the Infrared Array Camera during the Cryogenic Mission of the Spitzer Space Telescope
}

Carl J. Grillmair ${ }^{\mathrm{a}}$, Patrick J. Lowrance ${ }^{\mathrm{a}}$, Sean J. Carey ${ }^{\mathrm{a}}$, John R. Stauffer ${ }^{\mathrm{a}}$, James G. Ingalls ${ }^{\mathrm{a}}$, Jessica E. Krick ${ }^{\mathrm{a}}$, William J. Glaccum ${ }^{\mathrm{a}}$, Seppo Laine ${ }^{\mathrm{a}}$, Roberta Paladini ${ }^{\mathrm{a}}$, David L. Shupe ${ }^{\mathrm{a}}$, and Schuyler D. Van Dyk ${ }^{\mathrm{a}}$

${ }^{a}$ IPAC/Spitzer Science Center, California Institute of Technology, Mail Stop 314-6, 1200 E. California Blvd., Pasadena, CA, USA 91125

\begin{abstract}
We describe our ongoing efforts to model the field distortions of the Infrared Array Camera (IRAC) during the cryogenic portion of the Spitzer Space Telescope's operations. We have compared over two million measured source positions in $\sim 35,000$ IRAC images with their positions in Gaia Data Release 1 . Fitting $3^{\text {rd }}$ and $5^{\text {th }}$ order polynomials to the measured offsets, we find systematic uncertainties in IRAC-measured positions that are in the 50-60 milliarcsecond range for the 3.6 micron array, and 120-150 milliarcsecond range for the 4.5 micron array. A $5^{\text {th }}$-order fit does not appear to significantly improve the results over a $3^{\text {rd }}$ order fit. However, this may be due at least partly to the failure of our current centroiding technique to account for variations in the Point Response Functions across each detector. We anticipate making several improvements in our continuing analysis, including (i) the refitting of the positions and position angles of each IRAC image using the Gaia catalog, (ii) making use of a less position-sensitive centroiding algorithm, (iii) correcting where possible for the proper motions of detected sources, and (iv) significantly increasing the number of source position measurements. Once finalized, the resulting distortion corrections will be incorporated into the headers of the archived images.
\end{abstract}

Keywords: Spitzer Space Telescope

\section{INTRODUCTION}

Accurate astrometry is important in many different areas of astronomical research, from comparing the distributions of stars and dust in distant galaxies, to measuring distances and space motions of faint brown dwarfs in the solar neighborhood. By their nature, all telescope designs introduce optical distortions that alter both the apparent shapes and the positions of sources across the field of view. For the Infrared Array Camera (IRAC) arrays on the Spitzer Space Telescope, this distortion has been modeled using the SIP (Simple Imaging Polynomial) convention ${ }^{1}$. While the astrometric distortion correction used for the IRAC arrays during the cryogenic mission currently employs a $3^{\text {rd }}$ order polynomial, the correction for the warm mission has recently been refined and updated to use a $5^{\text {th }}$ order polynomial ${ }^{2}$. One motivation for the present study is to determine whether the cryogenic data would also benefit from a $5^{\text {th }}$ or higher order distortion correction. As there are now observations extending back over 14 years, it would clearly be useful to reduce the positional uncertainties at both ends of the time baseline. With the end of the Spitzer mission drawing near, and with the recent releases of the very high accuracy Gaia astrometric catalogs ${ }^{3,4}$, this appears to be an opportune time to improve Spitzer astrometry to the greatest extent possible.

\section{METHOD}

\subsection{Input Data}

We use nearly 35,000 IRAC images taken in the course of several large mapping programs executed during the Spitzer cryogenic mission (2003-2009). These include the Spitzer Galactic First Look survey, as well several thousand darkcalibration images of a field near the north ecliptic pole. For each image, we use the IDL implementation of DAOPHOT

Space Telescopes and Instrumentation 2018: Optical, Infrared, and Millimeter Wave, edited by Makenzie Lystrup,

Howard A. MacEwen, Giovanni G. Fazio, Proc. of SPIE Vol. 10698, 106985D

(C) 2018 SPIE · CCC code: 0277-786X/18/\$18 - doi: 10.1117/12.2313706 
FIND $^{5}$ to detect sources at the 5-sigma level or higher. These sources are then centroided using box_centroider, a simple first-moment centroiding algorithm. We initially chose this method as it appears to be less biased by intrapixel sensitivity variations than most other centroiding algorithms available within IDL. The resulting IRAC positions are then matched against the Gaia Data Release 1 catalog $^{3}$ using a 3 arcsec matching radius. To prevent multiple matches and erroneous offset determinations in particularly crowded fields such as globular clusters or the Galactic bulge, only sources that match exactly one Gaia source within this radius are included in our analysis.

One potential issue with our matched catalog is the inclusion of resolved galaxies. Such objects would have relatively poor and possibly color-dependent centroid computations and would therefore increase the noise in our final distortion maps. Since we are dealing with tens of thousands of images taken over many hundreds of square degrees of sky, and since IRAC's spatial resolution is relatively modest, it is neither practical nor useful to examine each image by eye. We rely instead on four mitigating factors: (i) Using the SHARP and ROUND parameters, the FIND algorithm rejects sources that are (a) significantly sharper (cosmic rays) or more extended (galaxies) than the stellar point spread function, or (b) significantly more extended in either the x- or y-pixel directions. (ii) We reject sources with box_centroider positional uncertainties that exceed 0.2 pixels. (iii) We also reject sources whose positional uncertainties in the Gaia catalog exceed 2 mas. (iv) Finally, for each pixel, we measure the mean IRAC-Gaia offset and remove all measurements that differ from this mean by more than 2.5 sigma. This sigma clipping is carried out twice. Spot checks of obvious galaxies in a few images show that application of these filters removes most if not all such objects from our final catalog. Gaia-matching, removal of objects with high positional uncertainties, and sigma-clipping reduce the total number of positional measurements in channel 1 from 3.7 million to roughly 2.1 million.

Another issue that became apparent during our analysis is that the pointing refinement used by the Spitzer Science Center pipeline to determine the sky position and rotation angle of each image is not perfect and can contribute significantly to the scatter. The pipeline uses available 2MASS sources in each field to adjust the zeropoints CRVAL1 and CRVAL2, as well as the CD rotation matrix. However, due to a limited number of sources per field (along with secondary effects such as source confusion in crowded fields and high proper motion objects) this pointing refinement is never perfect. Figure 1 shows median X-pixel shifts between sources found in both the Spitzer images and Gaia DR1 in the central regions of 28 images taken at various dithered locations in the globular cluster 47 Tuc. There are clear discontinuities between exposures that, if not corrected, would contribute significantly to the noise in our analysis. The RMS in these offsets is $\sim 0.15$ arcsec in channel 1 and $\sim 0.13 \operatorname{arcsec}$ in channel 2 . To reduce the contributions to the noise by errors in the pipeline pointing refinement, we compute and subtract the median $\mathrm{x}$ and y offsets between the Spitzer and Gaia positions in all case where there are more than 50 sources available to compute them. We use only sources falling in the central regions of the detectors $(64<\mathrm{x}<192,64<\mathrm{y}<192)$ where field distortions are minimal. We note that, if CRVAL1 and CRVAL2 are in error, the position angles determined by the pointing refinement module are almost certainly in error as well. While we have not attempted to refit the CD matrices in our present analysis, this will need to be done if we are to make full use of the Gaia measurements.

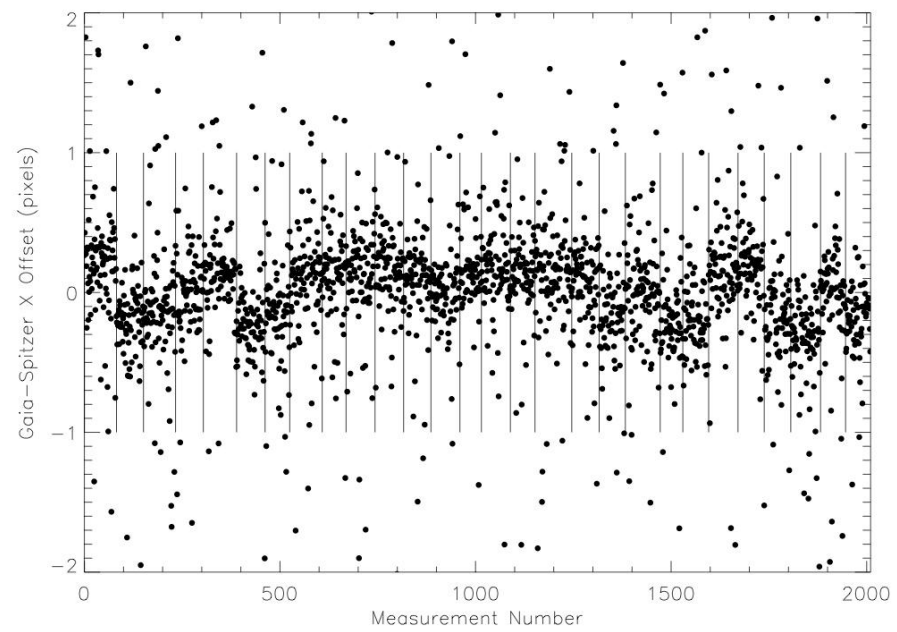

Figure 1. X-pixel offsets, before distortion correction, between Gaia DR1 and Spitzer [3.6] for centrally located sources in 28 dithered exposures of the globular cluster 47 Tuc. The vertical lines separate measurements in different exposures.

There are clear discontinuities and sometimes substantial offsets between successive exposures, illustrating the limits of the Spitzer pointing refinement pipeline, particularly in crowded fields. 
In Figures 2 and 3 we show the average deviations per pixel between the Gaia and IRAC positions after applying the Gaia-Spitzer offsets above but before making any distortion corrections. The mean number of measurements per pixel is $32 \pm 9$ for the 3.6 micron array, and (currently) about $12 \pm 5$ for the 4.5 micron array.

[3.6]

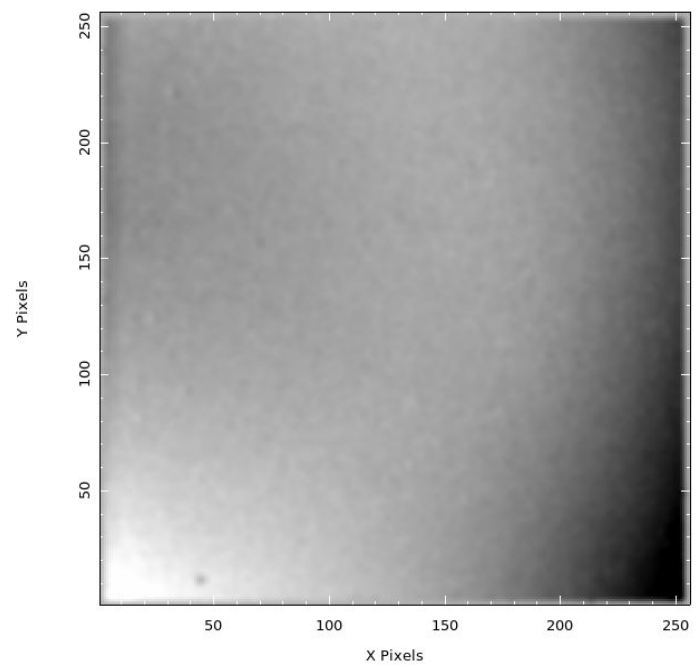

[4.5]

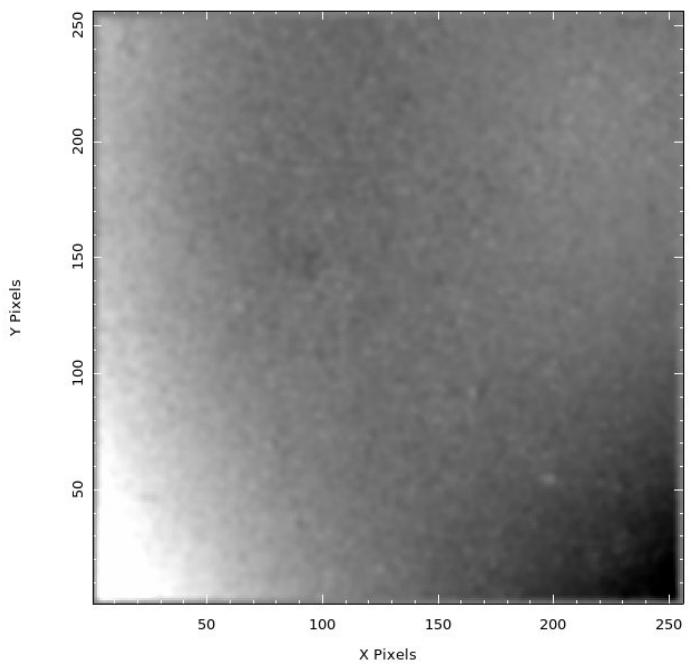

Figure 2. Mean x-pixel offsets as a function of array position for IRAC [3.6] and [4.5] during the cryo mission. Each pixel is a 2.5 sigma, twice-clipped mean of roughly 32 measurements for [3.6], and roughly 12 measurements for [4.5]. The gray scales extend from -1.5 arcsec (black) to 1.0 arcsec (white) for [3.6], and from -1 arcsec (black) to +1.6 arcsec (white) for [4.5].
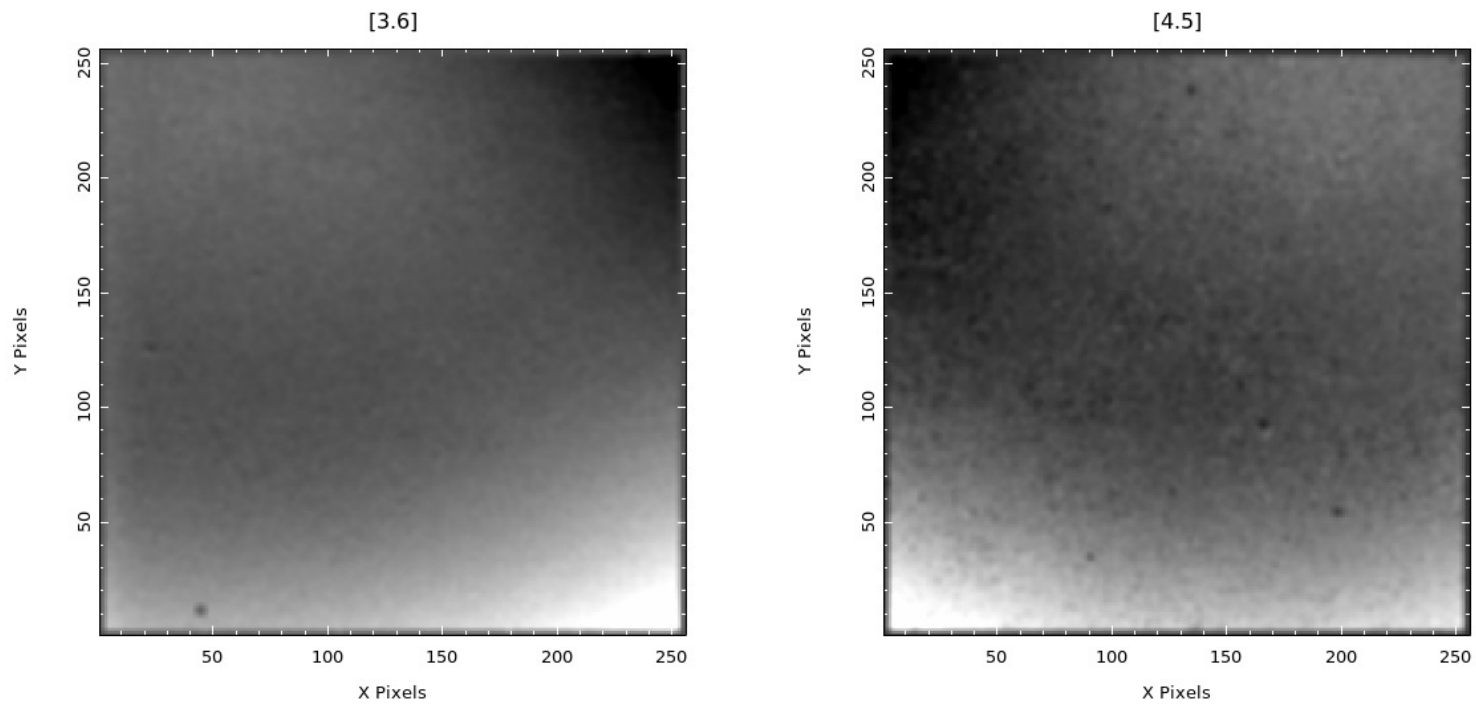

Figure 3. Same as Figure 1, but showing clipped mean y-pixel offsets as a function of array position. 


\subsection{Distortion Fitting}

While Figures 2 and 3 show the binned averages, our distortion fitting is carried out on the raw (unbinned) measurements. We use the IDL routine CURVEFIT, which employs a gradient-expansion algorithm to compute a nonlinear least-squares fit to an arbitrary function. Following the SIP (Simple Imaging Polynomial) convention ${ }^{1}$ adopted for Spitzer imaging we fit the measured offsets using a two-dimensional polynomial. The independent variables are the IRAC x- and y-pixel centroids while the dependent variables are the Gaia positions, transformed to IRAC pixel space using only the frame-center coordinates (CRVAL1, CRVAL2), rotation matrix, and a scale factor of 1.223 arcsec per pixel. Figures 4 and 5 show the resulting fits, along with the current cryogenic distortion correction.
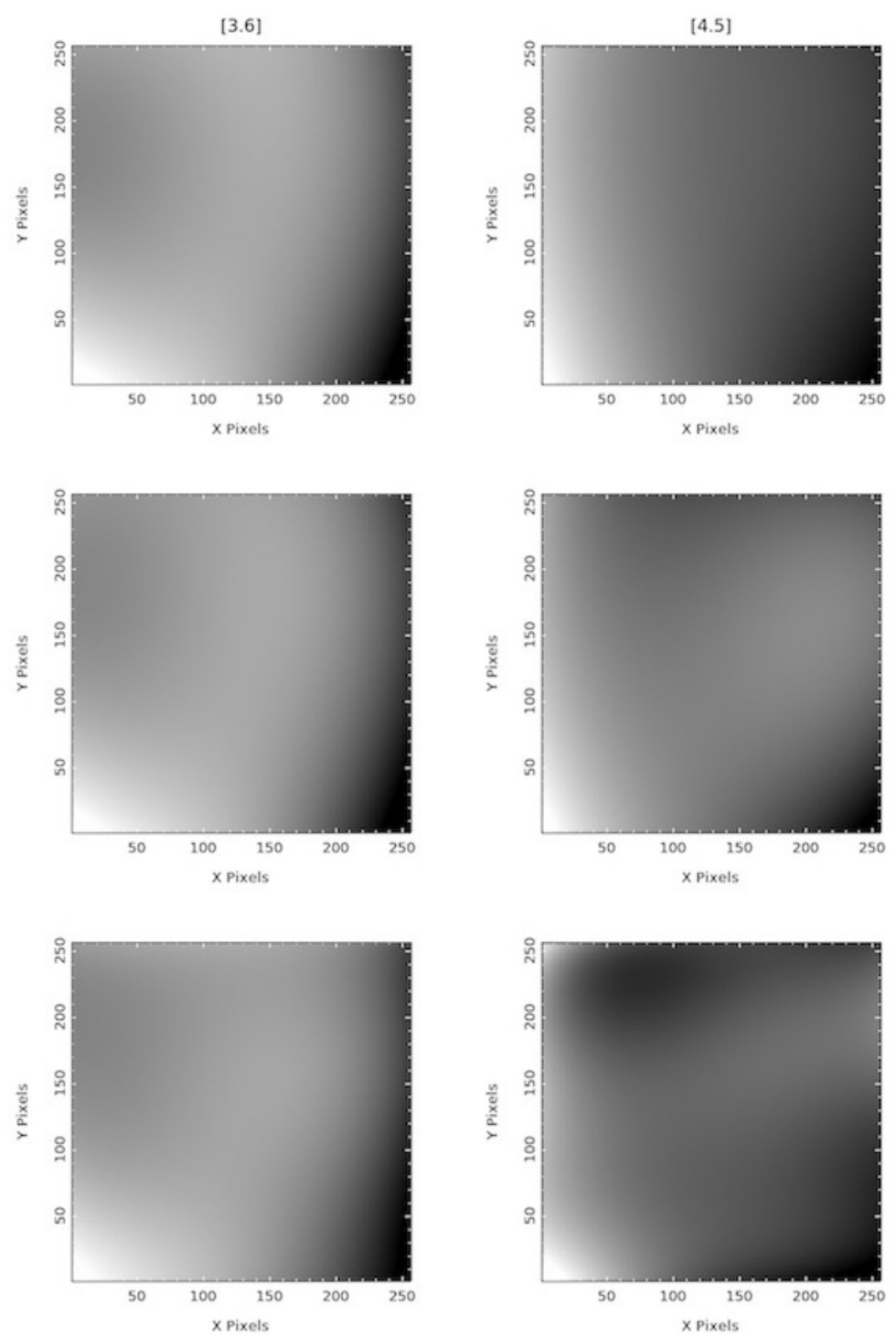

Figure 4. Polynomial fits to the mean x-pixel offsets in Figure 1. The upper row shows the $3^{\text {rd }}$-order model currently used in the Spitzer pipeline. The middle row shows new $3^{\text {rd }}$ order solutions derived from the data in Figures 2 . The bottom row shows fits using $5^{\text {th }}$ order polynomials. The stretch is linear and on the same scale as Figures 2 and 3. 

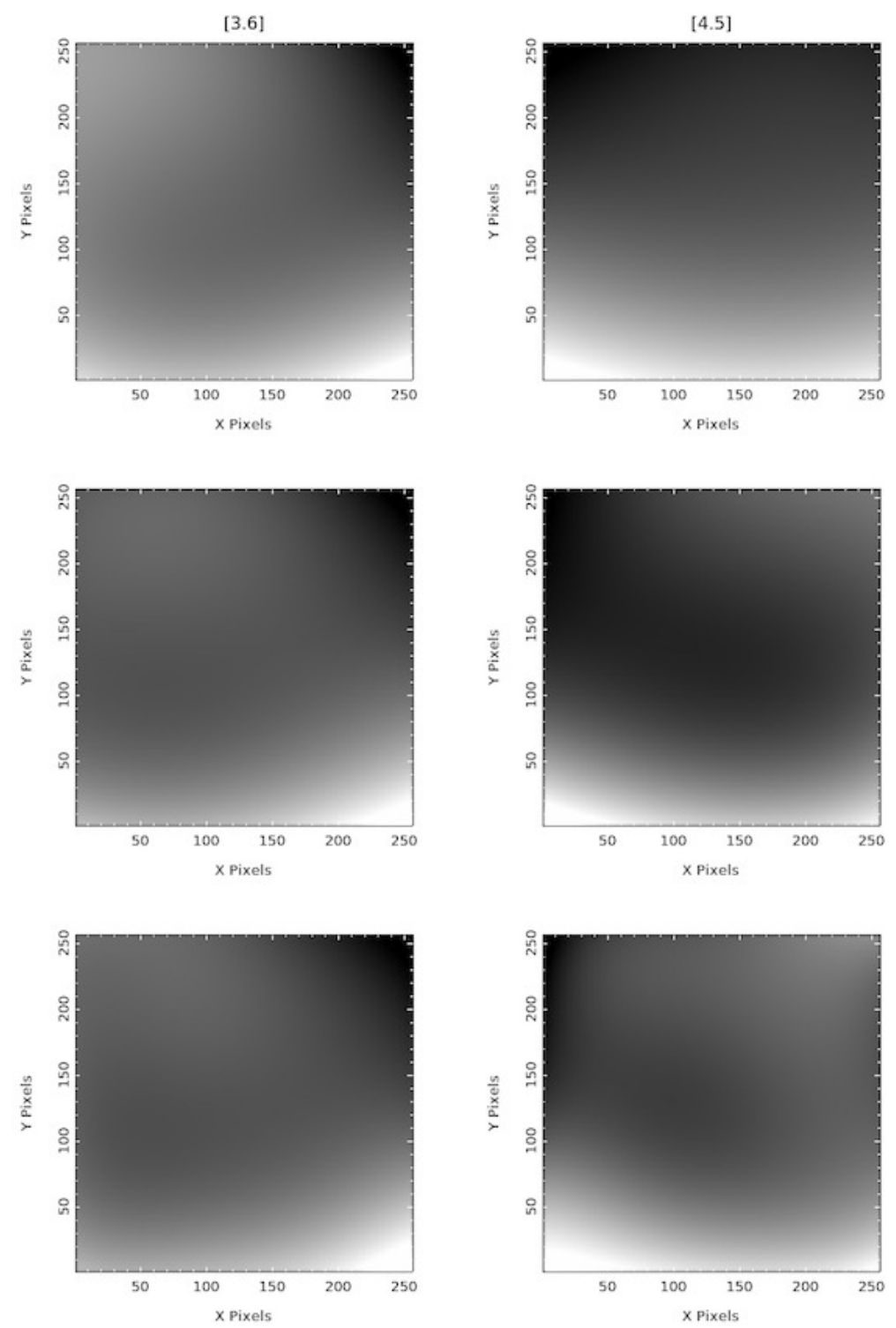

Figure 5. As in Figure 4, but for fits to the mean y-pixel offsets shown in Figure 3.

The orientations of the distortion corrections as a function of pixel position are shown in Figure 6. 

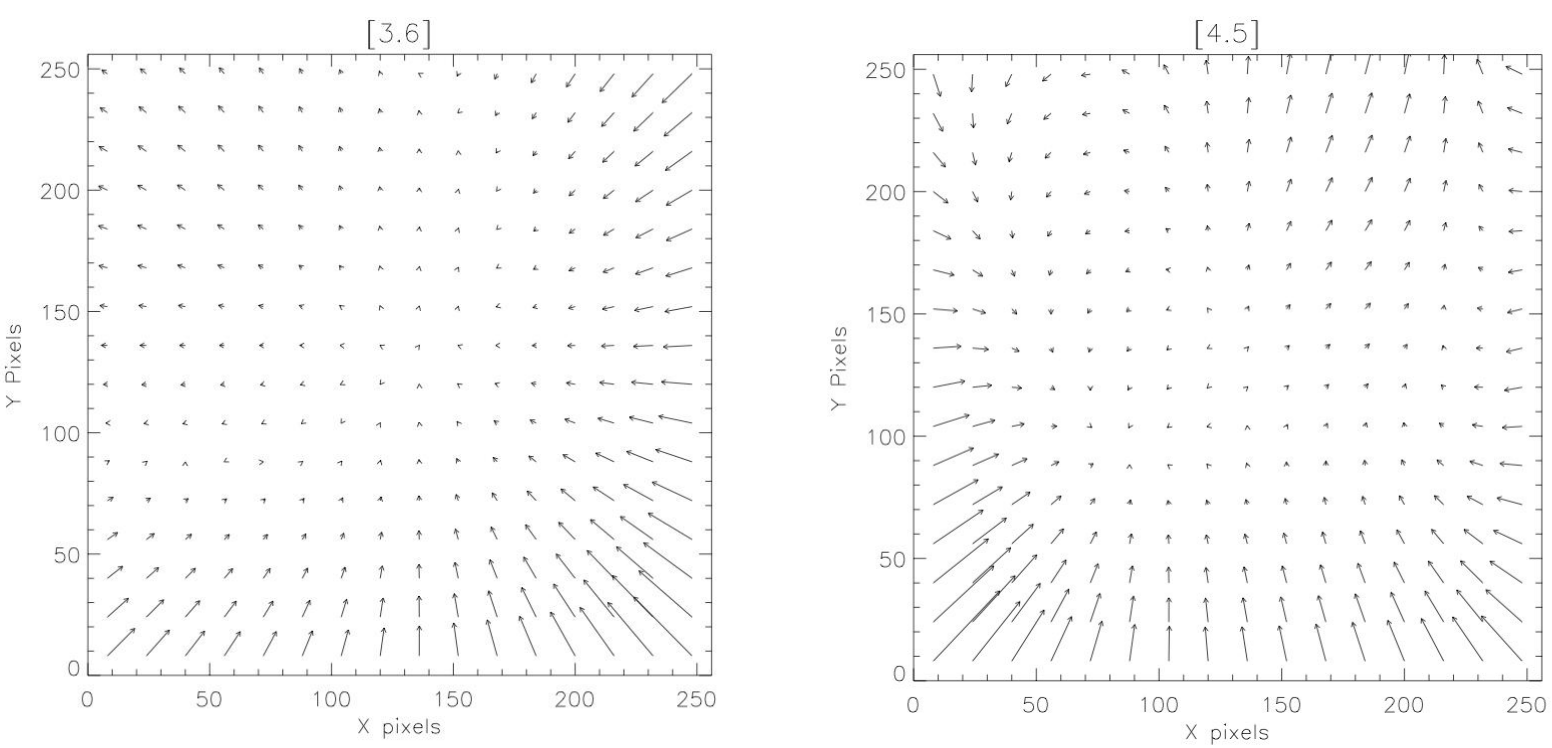

Figure 6. Deviations from a uniform plate scale using our $3^{\text {rd }}$ order distortion fit. The vectors have been lengthened by a factor of 20 to make them visible at this scale.

\section{RESULTS}

Comparing the fits in Figures 4 and 5 with the measured offsets in Figures 2 and 3, we see that our new fits match the observed undulations quite well.

Are the new fits better than the existing distortion corrections? In Figure 5 we show plots of the Allan variance as a function of spatial binning (frequency). This figure shows that the new $3^{\text {rd }}$ and $5^{\text {th }}$ order fits do indeed better model the astrometric distortions than do the corrections currently in the pipeline, particularly for the y-offsets. At the single pixel scale, the y-offset RMS in channel 1 is roughly halved, from $\sim 110$ mas to $\sim 53$ mas. For the $\mathrm{x}$ offsets the RMS is only slightly reduced, from 60 mas to $\sim 50$ mas. For channel 2 the RMS measured using the pipeline correction is 404 and 443 mas in $\mathrm{x}$ - and y-offsets, respectively. A new $3^{\text {rd }}$ order fit reduces this to 156 and 144 mas, respectively.

Are $5^{\text {th }}$ order distortion corrections measurably better than $3^{\text {rd }}$ order corrections? Comparing red and blue lines in Figure 7 shows that $5^{\text {th }}$ order fits do not significantly improve the variances, either at the pixel scale or at lower frequencies.
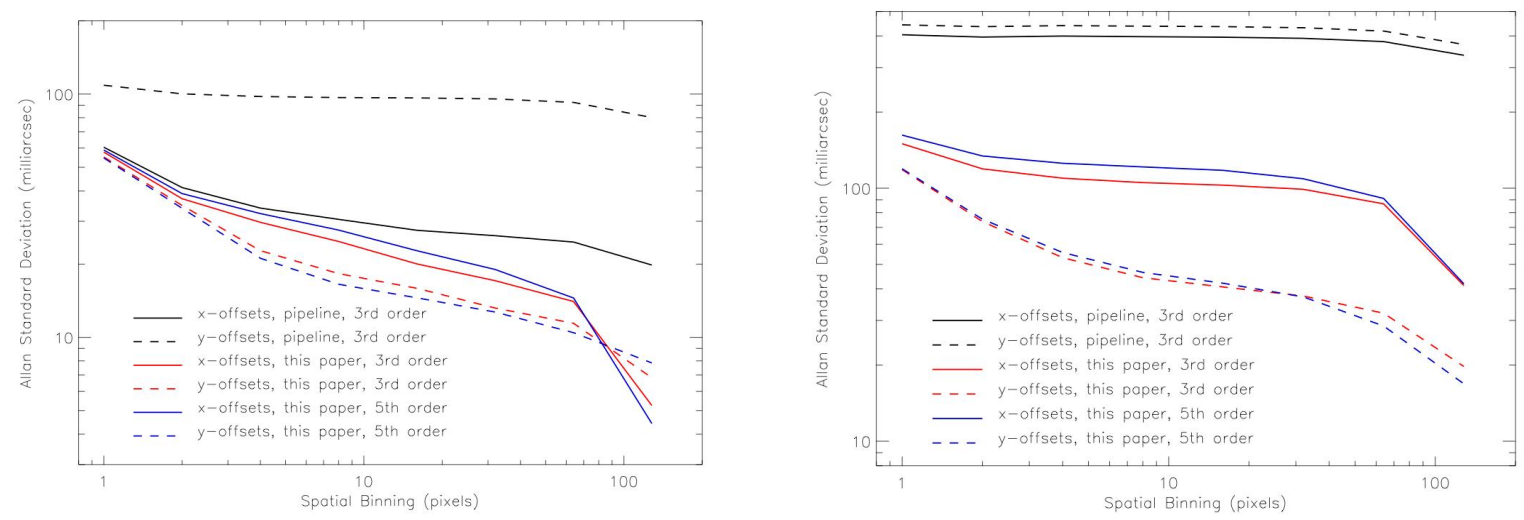

Figure 7. Allan variances for the pipeline distortion corrections as well as for $3^{\text {rd }}$ and $5^{\text {th }}$ order fits to the data in Figures 2 and 3. The left panel shows the results for 3.6 microns while the right panel shows the results for 4.5 microns. 
One important caveat concerns our use of a first-moment centroiding algorithm. Such an algorithm will not give consistent results if the underlying PRF is changing with position on the array. To estimate the size of this effect, we generated a grid of simulated PRFs and then used box_centroider to compute centroids. This exercise showed that the variable PRF can introduce centroid differences on the order of 100 mas from one side of the array to the other. This may explain the rather large standard deviations measured for the current pipeline distortion corrections (which were derived using APEX PRF-fitting centroids ${ }^{6}$ ) in Figure 7. It may also be partly responsible for the negligible improvement we find using a $5^{\text {th }}$ order distortion correction.

The next steps in our analysis will include going back and refitting the zero points CRVAL1 and CRVAL2, along with the CD matrices, for each IRAC image using the Gaia measurements. In addition, with the availability of Gaia Data Release 2, and since most of the cryo-mission IRAC images were taken more than a decade before the Gaia DR2 epoch, it should be straightforward to translate the Gaia source positions back to the epoch of each Spitzer observation. In view of our findings concerning box centroider, we also plan to move to $\mathrm{APEX}^{6}$ for source detection and centroiding. APEX accounts for changes in the PRF across the field, producing more consistent and less position-sensitive centroids. APEX has also been shown to better account for variations in intrapixel sensitivity and has enabled very high-order distortion corrections by others ${ }^{7}$. Finally, we anticipate expanding the number of input IRAC images (and therefore the number of positional measurements) by as much as a factor to two, particularly for channel 2 . In addition to reducing our uncertainties, this may enable us to test the suggestion that the field distortions are time dependent ${ }^{7}$.

\section{REFERENCES}

[1] Shupe, David. L, Moshir, M., Li, J., Makovoz, D., Narron, R., and Hook, R. N., "The SIP Convention for Representing Distortion in FITS Image Headers", in ASP Conference Series 347, [Astronomical Data Analysis Software and Systems XIV], ed. P. Shopbell, M. Britton, and R. Ebert, ASP, San Francisco, 491-495 (2005).

[2] Lowrance, Patrick, J, et al. "Enhancement of the Spitzer Infrared Array Camera (IRAC) distortion correction for parallax measurements," Proc. SPIE 9143, 58-64 (2014).

[3] Gaia Collaboration, Lindegren, L. et al., "Gaia Data Release 1. Astrometry: one billion positions, two million proper motions and parallaxes,", Astronomy and Astrophysics, 595, 4-36 (2016).

[4] Gaia Collaboration, Brown, A. G. et al, "Gaia Data Release 2. Summary of the contents and survey properties," Astronomy and Astrophysics, in press (2018).

[5] Stetson, Peter, B., "DAOPHOT - A computer program for crowded-field stellar photometry,", Pub. Astron. Soc. of the Pacific, 99, 191-222 (1987).

[6] Makovoz, D., and Marleau, F. R., "Point-Source Extraction with MOPEX,", Pub. Astron. Soc. Of the Pacific, 117, 1113-1128 (2005).

[7] Esplin, T. L., and Luhman, K. L., "Measuring High-Precision Astrometry with the Infrared Array Camera on the Spitzer Space Telescope," The Astronomical Journal, 151, 9-16 (2016). 